\title{
Significance of CD10-positive clonal B cell populations identified by flow cytometry in histologically benign gastric biopsies
}

\author{
Won-Tak Choi ${ }^{1,2,3} \cdot$ Sindhu Cherian ${ }^{2} \cdot$ Lorinda Soma $^{2}$
}

Received: 1 December 2015 / Accepted: 22 February 2016 /Published online: 3 March 2016

(C) Springer-Verlag Berlin Heidelberg 2016

\begin{abstract}
Historically, the presence of clonal B cell populations has been associated with malignancy in lymphoid proliferations. However, several studies have demonstrated that clonal B cell populations can be present in histologically reactive settings, but this has not been described in gastric samples. These studies have primarily shown clonality using molecular methods, with only a few studies showing clonality by flow cytometry. We evaluated 16 histologically benign gastric biopsies with CD10+ B cell populations identified by flow cytometry (13 clonal, 3 with kappa skew) and reviewed the morphology, endoscopic findings, and clinical course. Although the presence of clonal populations initially raised a diagnostic possibility of lymphoma in each case, morphologic examination supported a non-neoplastic reactive process. The $\mathrm{CD} 10+\mathrm{B}$ cell populations typically demonstrated increased or slightly increased CD38 (88\%), increased or slightly increased CD20 (81\%), decreased surface light-chain expression (69\%), normal CD19 (94\%), and those that were studied $(n=12)$ were BCL-2 negative or indeterminate by flow cytometry. Polyclonal B cells were present, and the CD10+ B cell population comprised less than $50 \%$ of the $\mathrm{B}$ cells in all cases. The available clinical information revealed no evidence of lymphoma in all 16 cases (follow-up ranging from 2 months
\end{abstract}

Lorinda Soma

somal@uw.edu

1 Department of Pathology, University of Washington School of Medicine, Seattle, WA 98195, USA

2 Department of Laboratory Medicine, University of Washington School of Medicine, 1959 NE Pacific Street, Box 357110, Seattle, WA 98195, USA

3 Department of Pathology, University of California at San Francisco, San Francisco, CA 94143, USA to 10 years). The presence of a CD10+ $\mathrm{B}$ cell clone (or lightchain skew) by flow cytometry in a gastric biopsy is not specific for lymphoid neoplasia and should be interpreted in the context of other immunophenotypic features as well as clinical and morphologic data.

Keywords CD10 positive $\cdot$ Clonal B cell population $\cdot$ Flow cytometry $\cdot$ Gastric lymphoma $\cdot$ Reactive gastritis

\section{Introduction}

Follicular lymphoma (FL) rarely arises in the stomach, whereas marginal zone lymphoma (MZL) of mucosa-associated lymphoid tissue (MALT) type is the predominant low-grade B cell lymphoma affecting the stomach [1-4]. Gastric lymphomas may present with symptoms such as dyspepsia and upper gastrointestinal bleeding and endoscopically may appear as nodularity, mass-like lesions and/or mucosal ulcers associated with varying degrees of mucosal thickening and vascularity $[1,2,5]$. Despite its rarity, FL of the stomach may be a consideration in the differential diagnosis of atypical lymphoid infiltrates in gastric biopsies, particularly if flow cytometric studies demonstrate a monotypic or clonal $\mathrm{CD} 10+\mathrm{B}$ cell population [6]. Furthermore, reactive gastritis can mimic these low-grade lymphomas, creating diagnostic difficulty in correctly classifying atypical gastric lymphoid infiltrates. Early-stage MZL is typically treated with antibiotics (although may include radiation and chemotherapy), where FL patients would not be treated with antibiotics and may receive more aggressive therapy $[4,7,8]$. Although specific phenotypic (as defined by flow cytometry and immunohistochemistry (IHC)) and genotypic features can significantly increase the accuracy of lymphoma diagnosis, the morphologic assessment remains the cornerstone of diagnosis. 
Studies have demonstrated that clonal B cell populations can be identified not only in gastric lymphomas but also in reactive gastritis via immunophenotyping and/or molecular techniques [9-11], although some authors would argue otherwise [12]. Therefore, the usefulness of using molecular studies to identify monoclonal B cell populations in distinguishing benign lymphoid infiltrates from malignant lymphoid neoplasm may be limited. Similarly, Kussick et al. demonstrated that light-chain-restricted B cell populations of follicle center type (with low- to intermediate-level CD10 expression) can be identified by flow cytometry and/or molecular methods in histologically benign lymph node and tonsillectomy specimens [13]. However, there are no reports in the literature that have specifically addressed the detection of a CD10+ clonal $\mathrm{B}$ cell population by flow cytometry in histologically benign gastric biopsies and its potential implications.

In this regard, we describe 16 cases of histologically benign gastric biopsies that demonstrated $\mathrm{CD} 10+$ clonal (or light chain skewed) B cell populations identified by flow cytometry, illustrating the diagnostic complexities encountered when phenotypic abnormalities raise the question of neoplasia. In one of the cases studied, the population size by flow cytometry was large enough to raise a high level of concern for neoplasia (greater than $30 \%$ of white cells). Therefore, we sought to determine the clinical behavior of $\mathrm{CD} 10+$ clonal (or light chain skewed) $\mathrm{B}$ cell populations seen in the stomach and to identify the morphologic, flow cytometric, and/or endoscopic findings that can help distinguish reactive CD10+ clonal/ skewed B cell populations from bona fide lymphoid neoplasms (including MZL and FL).

\section{Materials and methods}

\section{Patients and data collection}

Using our pathology information system (Power Path) and Hematopathology flow cytometry database, 16 histologically benign gastric biopsies that contained $\mathrm{CD} 10+$ clonal/skewed B cell populations were identified at the University of Washington Hematopathology Laboratory between 2000 and 2014. Hospital electronic medical records were retrospectively reviewed for these patients to collect additional clinical information, including prior history of lymphoma, endoscopic findings, follow-up gastric biopsies (or lack thereof), possible causes of gastritis (such as Helicobacter pylori infection), and if there was evidence of new or recurrent lymphoma in the available clinical history. As comparison cohorts, the gastric biopsies with $\mathrm{CD} 10+$ clonal/skewed $\mathrm{B}$ cell populations were compared to bona fide low-grade B cell lymphomas with flow cytometry (including ten MZLs of the stomach and seven FLs of MALT sites). Our study was approved by the University of
Washington Institutional Review Board for human subjects research, \#48070.

\section{Flow cytometry}

Fresh tissue was collected in Roswell Park Memorial Institute (RPMI) medium and processed for flow cytometry as described elsewhere [13]. Flow cytometry was performed as part of the clinical evaluation, using either a modified four-laser, ten-color Becton Dickenson LSR II flow cytometry (BD Biosciences, San Jose, CA) and prior to 2005 either a fourcolor FC-500 or Coulter Epics XL instrument (Beckman Coulter, Brea, CA). The antibody panels performed are listed in Table 1. At least 7000 events were acquired for the B cell analysis in all cases, with $>20,000$ events acquired in the majority. Data compensation and analysis were performed using software developed in-house (WoodList version 2.7 developed by Dr. Brent Wood, University of Washington Medical Center). Flow cytometric data was independently analyzed by two hematopathologists (L.S. and S.C.) to retrieve pertinent data, including the expression of light chain (including intensity and kappa/lambda ratio) as well as expression and intensity of CD5, CD10, CD19, CD20, CD38, and BCL-2. Percentage of $\mathrm{CD} 10+\mathrm{B}$ cells (as compared to the numbers of total B cells or white cells) was also assessed. Flow cytometric evidence of clonality was determined using criteria reported by others (reactive germinal centers (GCs) showing a kappa/lambda ratio of 1.1-3.07) [14]; however, given the lack of consensus and reported variation [15], we opted to use a kappa/lambda ratio of $\leq 0.3$ and $\geq 5$ as evidence of clonality and $0.31-0.5$ and 3.5-4.9 as evidence of skew. Antigenic intensity on the population of interest was assessed in comparison to the associated polyclonal non-GC B cells in the sample using visual inspection of the mean. Markers were considered slightly increased if the mean of the population in question was greater than the mean of the associated polyclonal B cells, but the populations were still overlapping and increased if there was no significant overlap (with the same criteria for decreases in intensity). Light chain was considered dim if the visual mean of the $\mathrm{CD} 10+$ population was less than that of the polyclonal, CD10-negative B cells. BCL-2 was considered positive if the population clearly showed greater intensity than the associated CD10-negative polyclonal B cells and T cells.

\section{Morphology}

The gastric biopsies were independently reviewed by two hematopathologists (L.S. and S.C.) to confirm the histological diagnosis and identify pertinent histological characteristics, including the presence of GCs, lymphoepithelial lesions (LEL), and the degree (mild, moderate, or severe) and pattern (diffuse, aggregate/follicular, or both) of lymphocytic 
Table 1 Flow cytometry panel: antibodies, fluorochromes, and vendor

B cell panel CD5: PE-Cy5.5 CD10: APC $^{\mathrm{b}}$ CD19: PE-Cy7 CD20: V450 $^{\mathrm{a}}$ CD38: A594 ${ }^{\mathrm{a}}$ CD45: APC-H7 Kappa: FITC $^{\mathrm{a}} \quad$ Lambda: PE $^{\mathrm{a}}$

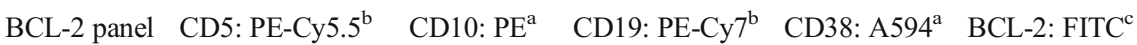

Note that hyphenated fluorochromes are tandem conjugates

FITC fluorescein isothiocyanate, $P E$ phycoerythrin, $C y$ cyanine dye, $A P C$ allophycocyanin, $A P C-H 7$ allophycocyanin-hilite $7, V 450 \mathrm{Violet} 450 \mathrm{~nm}$, A594 Alexa $594 \mathrm{~nm}$

${ }^{\mathrm{a}}$ Vendor: Becton Dickenson

${ }^{\mathrm{b}}$ Vendor: Beckman Coulter

${ }^{\mathrm{c}}$ Vendor: DakoCytomation

infiltrates. Multiple hematoxylin and eosin (H\&E) levels were evaluated in all cases, and IHC studies were performed as part of the diagnostic work-up in some of the cases (IHC evaluated but not reported).

\section{Results}

\section{Flow cytometric characteristics of gastric CD10+ clonal B cell populations and correlation with morphological and endoscopic findings}

All 16 cases (13 patients) in this series are summarized in Table 2. The gastric biopsies came from five males and eight females ranging in age from 23 to 71 years (mean, 42 and 50 years, respectively). H. pylori infection was detected in Giemsa and H\&E stains in 5 (31\%) of the 16 cases. Drug and infection were listed as possible etiologies of gastritis in the remaining cases, without histological evidence of $H$. pylori. Four patients had a prior history of lymphoma (two with gastric MZL, one with splenic MZL, and one with gastric large B cell lymphoma (LBL)). Both gastric MZL cases had no flow studies but were both CD10 and BCL-6 negative by IHC. The splenic MZL was CD10 negative by flow cytometry, and the gastric LBL was reported from an outside institution without mention of flow cytometry or GC marker expression. In 13 cases, a surface light-chain-restricted B cell population was identified in gastric biopsies, with three cases demonstrating a light-chain skew (using the previously stated criteria for clonality). Eleven (69\%) of the 16 cases demonstrated kappa light-chain restriction or skew, whereas the remaining five cases (31\%) showed lambda light-chain restriction or skew. A majority of cases (69\%) showed dim intensity of light-chain expression as compared to background non-GC $\mathrm{B}$ cells, as described by others in reactive scenarios $[14,16]$. The mean percentage of clonal/skewed CD10+ B cells was estimated to be $16.2 \%(\min =4.2 \%, \max =47 \%)$ among all $\mathrm{B}$ cells and $6.3 \%(\min =0.3 \%, \max =34.3 \%)$ among all white cells. All light-chain-restricted/skewed B cell populations demonstrated the characteristic follicle-center-

Table 2 Flow cytometric and morphological findings of CD10+ clonal B cell populations in histologically benign gastric biopsies

\begin{tabular}{|c|c|c|c|c|c|c|c|c|c|c|c|c|}
\hline $\begin{array}{l}\text { Case } \\
\text { no. }\end{array}$ & $\begin{array}{l}\text { Age/sex } \\
\text { (follow-up) }\end{array}$ & $\begin{array}{l}\text { B cell } \kappa / \\
\lambda \text { ratio }\end{array}$ & $\begin{array}{l}\text { BCL- } \\
2\end{array}$ & CD19 & $\mathrm{CD} 20$ & $\mathrm{CD} 38$ & $\begin{array}{l}\text { Estimated clonal/ } \\
\text { skewed B cells } \\
\text { (\% of B cells) }\end{array}$ & $\begin{array}{l}\text { Estimated clonal } \\
\text { B cells (\% } \\
\text { of white cells) }\end{array}$ & $\mathrm{GC}$ & LEL & $\begin{array}{l}\text { Degree of } \\
\text { infiltration }\end{array}$ & $\begin{array}{l}\text { Pattern of } \\
\text { infiltration }\end{array}$ \\
\hline 1 & $46 / F$ (2 months) & $\kappa(\operatorname{dim}, 3.8)$ & Neg & Normal & Normal & Increased & 10.6 & 2 & Yes & No & Mild & Aggregate \\
\hline 2 & $63 / \mathrm{F}$ (1 year) & $\kappa(147)$ & Neg & Normal & Increased & Increased & 37.9 & 17.1 & No & No & Mild & Aggregate \\
\hline 3 & 30/M (2 years) & $\kappa(\operatorname{dim}, 23)$ & Neg & Slightly increased & Increased & Increased & 44.4 & 4.4 & No & No & Mild & Aggregate and diffuse \\
\hline 4 & 61/F (2 years) & $\lambda(0.08)$ & ID & Normal & Normal & Normal & 4.3 & 1.1 & No & No & No & No \\
\hline 5 & 30/M (4 years) & $\lambda(0.04)$ & Neg & Normal & Slightly increased & Increased & 6.1 & 2.4 & Yes & No & Mild & Aggregate \\
\hline 6 & $33 / \mathrm{F}$ (5 months) & $\kappa(\operatorname{dim}, 15.8)$ & Neg & Normal & Increased & Increased & 12 & 4.2 & No & No & Mild & Aggregate \\
\hline 7 & 69/M (5 years) & $\kappa(\operatorname{dim}, 5.4)$ & TFE & Normal & Slightly increased & Increased & 7.2 & 3.4 & Yes & No & Mild & Aggregate \\
\hline 8 & 23/M (2 years) & $\lambda(\operatorname{dim}, 0.02)$ & Neg & Normal & Increased & Increased & 4.2 & 0.3 & No & No & Mild & Aggregate \\
\hline 9 & 59/M (1 year) & $\kappa(\operatorname{dim}, 12.5)$ & Neg & Normal & Normal & Increased & 12.2 & 5.9 & Yes & No & Moderate & Aggregate and diffuse \\
\hline 10 & $61 / F$ (6 years) & $\lambda(\operatorname{dim}, 0.4)$ & Neg & Normal & Increased & Increased & 15.4 & 8.3 & Yes & No & Moderate & Aggregate \\
\hline 11 & $58 / \mathrm{F}$ (1 year) & $\lambda(0.04)$ & ND & Normal & Slightly increased & Normal & 8.3 & 0.35 & No & No & Moderate & Aggregate and diffuse \\
\hline 12 & 71/F (10 years) & $\kappa(\operatorname{dim}, 10.1)$ & TFE & Normal & Slightly increased & Increased & 5.9 & 1.3 & No & No & Mild & Aggregate \\
\hline 13 & $57 / \mathrm{F}^{\mathrm{a}}(10$ years $)$ & $\kappa(\operatorname{dim}, 59)$ & Neg & Normal & Slightly increased & Increased & 10.6 & 0.7 & No & No & Mild & Aggregate \\
\hline 14 & $54 / \mathrm{F}^{\mathrm{a}}$ & $\kappa(\operatorname{dim}, 7.1)$ & Neg & Normal & Increased & Slightly increased & 16 & 8.8 & Yes & No & Moderate & Aggregate \\
\hline 15 & $54 / \mathrm{F}^{\mathrm{a}}$ & $\kappa(\operatorname{dim}, 3.9)$ & Neg & Normal & Slightly increased & Slightly increased & 16.8 & 5.7 & Yes & No & Moderate & Aggregate \\
\hline 16 & $54 / \mathrm{F}^{\mathrm{a}}$ & $\kappa(9.7)$ & ND & Normal & Increased & Increased & 47 & 34.3 & Yes & No & Moderate & Aggregate and diffuse \\
\hline
\end{tabular}

${ }^{a}$ The same patient with multiple cases/time points of assessment

$I D$ indeterminate, $N D$ not done, TFE two few events, Neg negative, GC germinal left, LEL lymphoepithelial lesion 
associated CD10 expression. In addition, as reported by others $[13,14,17,18]$, we noted more consistent, slightly increased to increased CD20 and increased CD38 expression in 13 $(81 \%)$ and $14(88 \%)$ of the 16 cases, respectively, when compared to the associated polyclonal CD10-negative B cells (Fig. 1a, b). Figure 2 additionally demonstrates cases with "normal" or "slightly increased" CD20 and/or CD38 expression in comparison to a case demonstrating "increased" CD20 and CD38 expression. The levels of CD19 in 15 (94\%) of the 16 clonal populations were similar to those of the background polyclonal B cells. There was no evidence of CD5 expression or BCL-2 overexpression in all cases examined (16 and 11 cases, respectively)

The morphologic examinations of all 16 cases showed mild to moderate lymphocytic infiltrates with a predominantly nodular architectural pattern in 15 of 16 (94\%), a subset of which showed an additional diffuse component (5 of 15; $33 \%$ ) (Fig. 3a-d). One case demonstrated no appreciable lymphoid infiltrate. There were no LELs in all cases. GCs were identified in $8(50 \%)$ of the 16 cases (histologically composed of polymorphous lymphoid cells with tingible body macrophages and mitotic activity, without follicular effacement by a uniform population of small cleaved cells). Upper endoscopy demonstrated mucosal thickening/irregularity and/ or erosions/ulcers in seven cases. Only one case had both mucosal ulcers and associated mucosa thickening, which are common gross findings in patients with gastric lymphomas [2]. No mass-like lesions were identified. No patients developed lymphoma based on the available clinical (and, in some cases, histologic) follow-up (clinical follow-up 2 months to 10 years with average follow-up of 3.4 years).

\section{Comparison of gastric CD10+ clonal B cell populations to histologically proven low-grade B cell lymphomas}

As comparison cohorts, the gastric biopsies with CD10+ clonal B cell populations were compared to bona fide low-grade B cell lymphomas (including ten gastric MZLs and seven FLs from non-gastric, MALT sites). All gastric MZL cases were clonal (70\% kappa restricted) without expression of CD10 or CD5 (Table 3), and six of the ten cases showed normal CD20 (three increased/slightly increased and one decreased). The
Fig. 1 Dot plots showing a reactive clonal $\mathrm{CD} 10+$ population $(\mathbf{a}, \mathbf{b})$ in comparison to follicular lymphoma at mucosal/extranodal sites $(\mathbf{c}, \mathbf{d}) . \mathrm{CD} 10+\mathrm{B}$ cells are highlighted in yellow (and emphasized if $<50 \%$ of the total $\mathrm{B}$ cells) and polyclonal B cells highlighted in blue. Notice the normal CD19 and increased CD20 and CD38 expression in the reactive clonal $\mathrm{CD} 10+\mathrm{B}$ cell populations (arrows, a, b) versus decreased CD19, normal or decreased CD20, and normal CD38 in the follicular lymphoma (arrows, c, d)

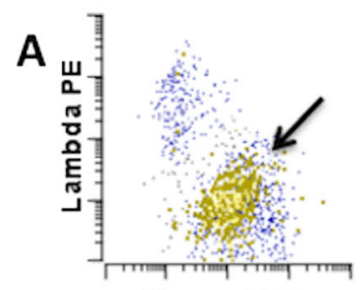

Kappa FITC

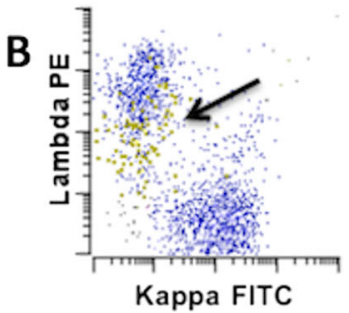

C
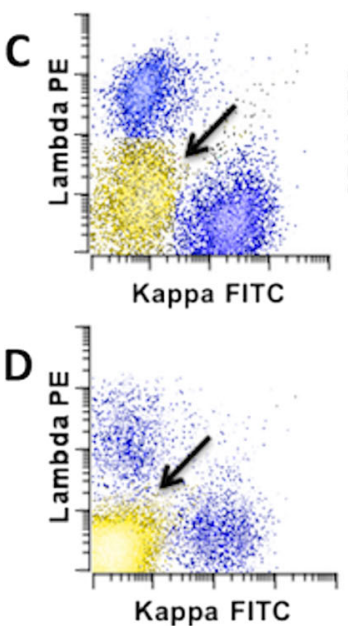

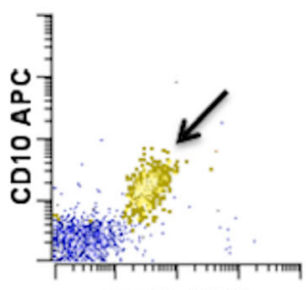

CD38 A594

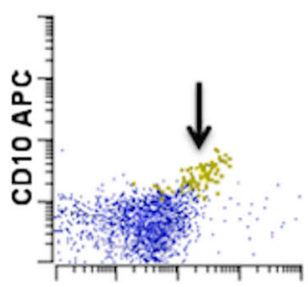

CD38 A594

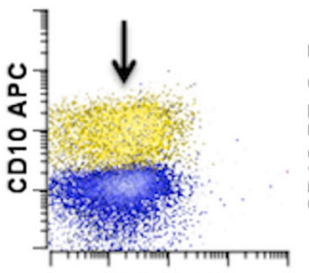

CD38 A594

CD38 A594

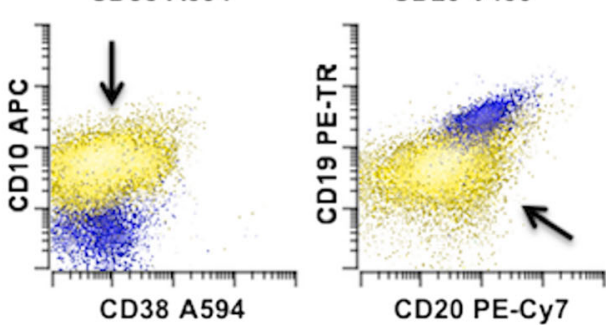

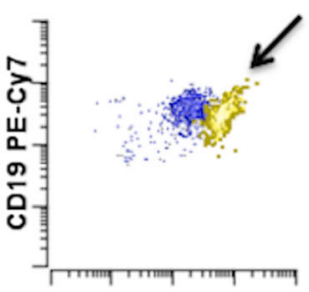

CD20 V450

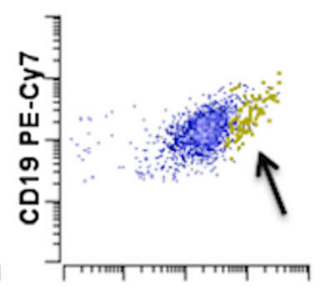

CD20 V450

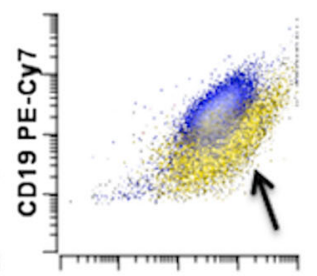

CD20 V450

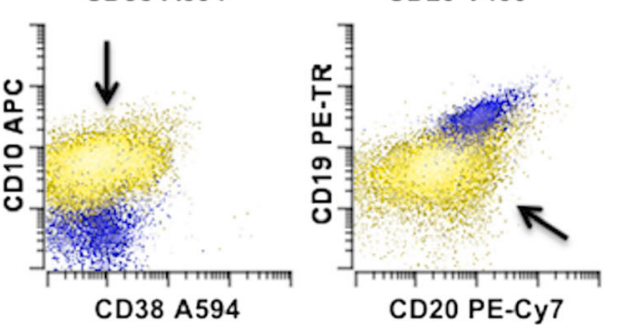

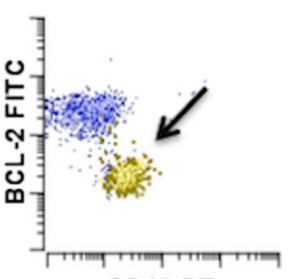

CD10 PE

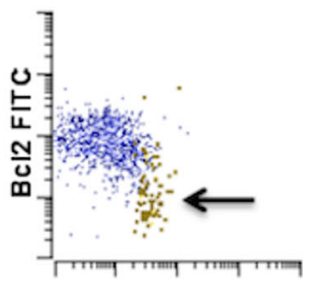

CD10 PE

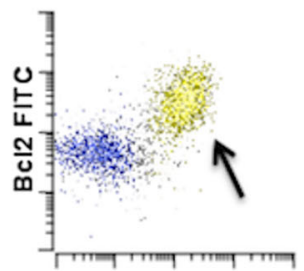

CD10 PE 
Fig. 2 Dot plots of all lymphocytes. The clonal or skewed CD10+ population is highlighted and emphasized in yellow (arrowed population); the CD19+, CD10-negative polyclonal B cells are highlighted in blue, and T cells are highlighted in green. The reactive, clonal $\mathrm{CD} 10+\mathrm{B}$ cell population with the typical increase in CD20 and CD38 expression (a, case 2 from Table 2) can be compared to a case with only slightly increased CD20 and CD38 (b, case 15), a case with slightly increased CD20 and normal CD38 (c, case 11), and a case with normal CD20 and increased CD38 (d, case 1). The antigens that were considered "slightly increased" show some overlap with the CD10-negative B cells, although show a slightly increased shift in antigen intensity

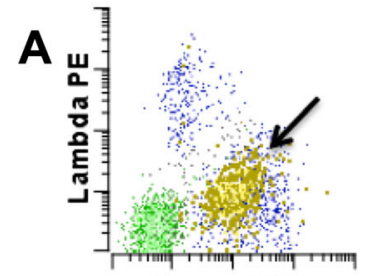

Kappa FITC

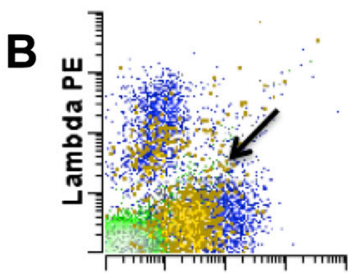

Kappa FITC

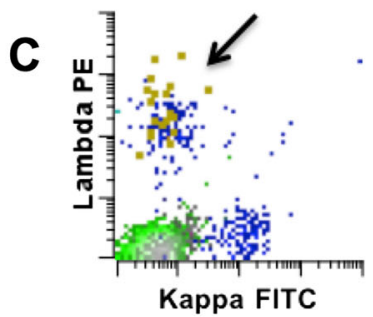

D

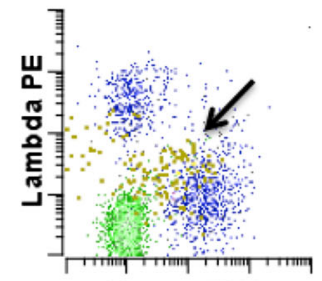

Kappa FITC
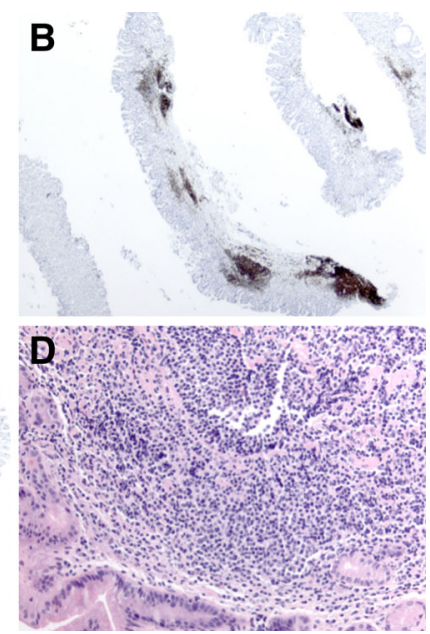
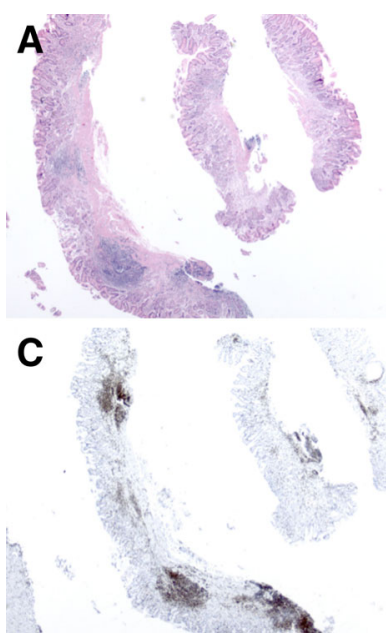

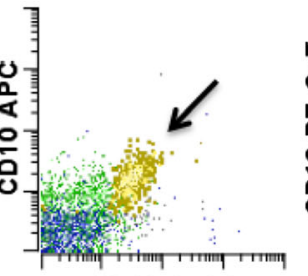

CD38 A594

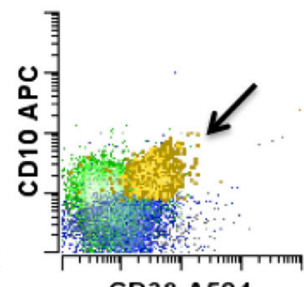

CD38 A594

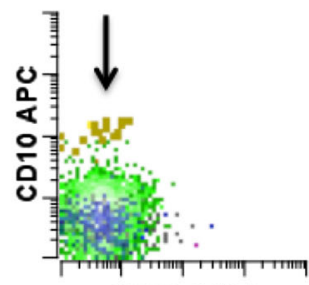

CD38 A594

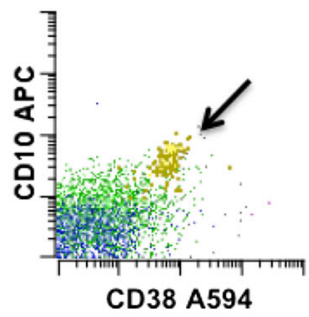

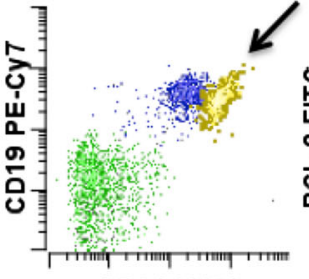

CD20 V450

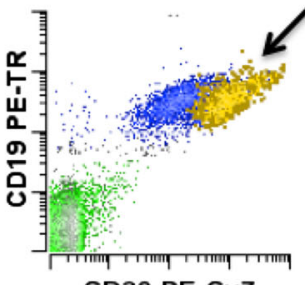

CD20 PE-Cy7
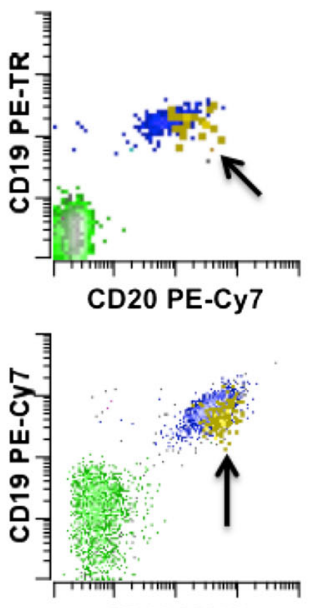

CD20 V450

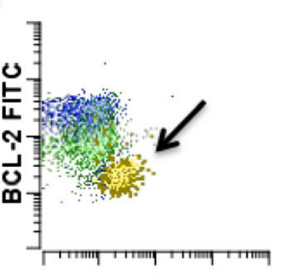

CD10 PE

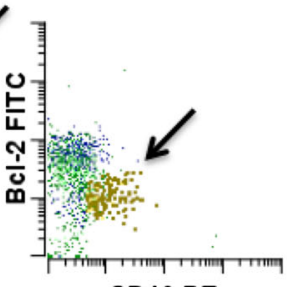

CD10 PE levels of CD19 and CD38 in all ten clonal populations were similar to those of the polyclonal B cells in the background (normal expression level). The mean percentage of the clonal population was relatively high in gastric MZLs, representing $84 \%(\min =60 \%, \max =98 \%)$ among all $\mathrm{B}$ cells and $40 \%$ $(\min =10 \%, \max =87 \%)$ among all white cells. Consistent with the greater mean percentage of clonal populations identified by flow cytometry, all cases showed moderate to severe lymphocytic infiltrates in a diffuse architectural pattern with the presence of LELs in nine $(90 \%)$ of the ten cases (Fig. $4 a-$ d). No GC was identified in nine $(90 \%)$ of the ten cases. Endoscopically, MZL showed mucosal thickening/erosion/ulcerations in $90 \%$ of the cases, with one of these patients demonstrating a mass-like, linitis plastica appearance.

Due to the rarity of gastric FL, seven FL cases from nongastric sites (including two duodenal, one lung, two parotid, and two orbital) were analyzed for comparison (Table 4). As expected, all FL cases were composed of clonal or light-chainnegative, $\mathrm{CD} 10+\mathrm{B}$ cell populations. The clonal populations demonstrated normal to decreased CD20 expression in six of seven cases (86\%), and CD19 was variable (three normal, three decreased, and one increased) (Fig. 1c, d). All cases
$\mathrm{CD} 10+$ population identified by flow cytometry (case 2 from Table 2). a A mild to moderate lymphocytic infiltrate is seen with a predominantly nodular architectural pattern $(H \& E$ stain, $\times 2)$. b CD20 stain highlights B cells $(\times 2)$, some areas more numerous than $\mathrm{CD} 3$. c CD3 stain highlights $\mathrm{T}$ cells, which are less numerous than the B cells, but not markedly less $(\times 2)$. d Lymphocytes in reactive gastritis tend to have round nuclear contours without abundant cytoplasm, and no lymphoepithelial lesions are seen $(H \& E, \times 20)$ 
Table 3 Flow cytometric and morphological findings of clonal B cell populations in gastric marginal zone lymphomas

\begin{tabular}{|c|c|c|c|c|c|c|c|c|c|c|c|c|}
\hline $\begin{array}{l}\text { Case } \\
\text { no. }\end{array}$ & $\begin{array}{l}\text { Age/ } \\
\text { sex }\end{array}$ & $\begin{array}{l}\mathrm{B} \text { cell } \kappa / \\
\lambda \text { ratio }\end{array}$ & $\mathrm{CD} 10$ & CD19 & $\mathrm{CD} 20$ & $\mathrm{CD} 38$ & $\begin{array}{l}\text { Estimated clonal B } \\
\text { cells ( } \% \text { of B cells) }\end{array}$ & $\begin{array}{l}\text { Estimated clonal B } \\
\text { cells ( } \% \text { of white cells) }\end{array}$ & GC & LEL & $\begin{array}{l}\text { Degree of } \\
\text { infiltration }\end{array}$ & $\begin{array}{l}\text { Pattern of } \\
\text { infiltration }\end{array}$ \\
\hline 1 & ${ }^{77 /} \mathrm{M}$ & $\lambda(0.03)$ & No & Normal & Increased & Normal & 88 & 55 & Yes & Yes & Severe & Diffuse \\
\hline 2 & ${ }_{\mathrm{M}}^{71 /}$ & $\kappa(138)$ & No & Normal & Normal & Normal & 70 & 20 & No & Yes & Severe & Diffuse \\
\hline 3 & ${ }^{79 /} \mathrm{M}$ & $\lambda(0.23)$ & No & Normal & Increased & Normal & 85 & 23.20 & No & No & Severe & Diffuse \\
\hline 4 & $64 / \mathrm{F}$ & $\lambda(0.035)$ & No & Normal & Normal & Normal & 95 & 12 & No & Yes & Severe & Diffuse \\
\hline 5 & ${ }_{\mathrm{M}}^{58 /}$ & $\kappa(5.6)$ & No & Normal & Decreased & Normal & 60 & 38.40 & No & Yes & Severe & Diffuse \\
\hline 6 & ${ }^{43 /} \mathrm{M}$ & $\kappa(32)$ & No & Normal & Normal & Normal & 91 & 56.40 & No & Yes & Moderate & Diffuse \\
\hline 7 & $37 / \mathrm{F}$ & $\kappa(14.5)$ & No & Normal & Normal & Normal & 81 & 65.30 & No & Yes & Severe & Diffuse \\
\hline 8 & $87 / \mathrm{F}$ & $\kappa(142)$ & No & Normal & Slightly increased & Normal & 98 & 87 & No & Yes & Severe & Diffuse \\
\hline 9 & $69 / \mathrm{F}$ & $\kappa(18)$ & No & Normal & Normal & Normal & 85 & 10.30 & No & Yes & Severe & Diffuse \\
\hline 10 & $35 / \mathrm{F}$ & $\kappa(82)$ & No & Normal & Normal & Normal & 86 & 28.10 & No & Yes & Severe & Diffuse \\
\hline
\end{tabular}

GC germinal left, LEL lymphoepithelial lesion

showed normal CD38 expression. These patterns of expression are similar to what has been reported previously $[18,19]$, including decreased expression of CD19 and normal expression of CD38 (decreased in comparison to follicular hyperplasia). The reported expression of CD20 in FL has been more variable. BCL-2 expression was assessed and positive in two cases.

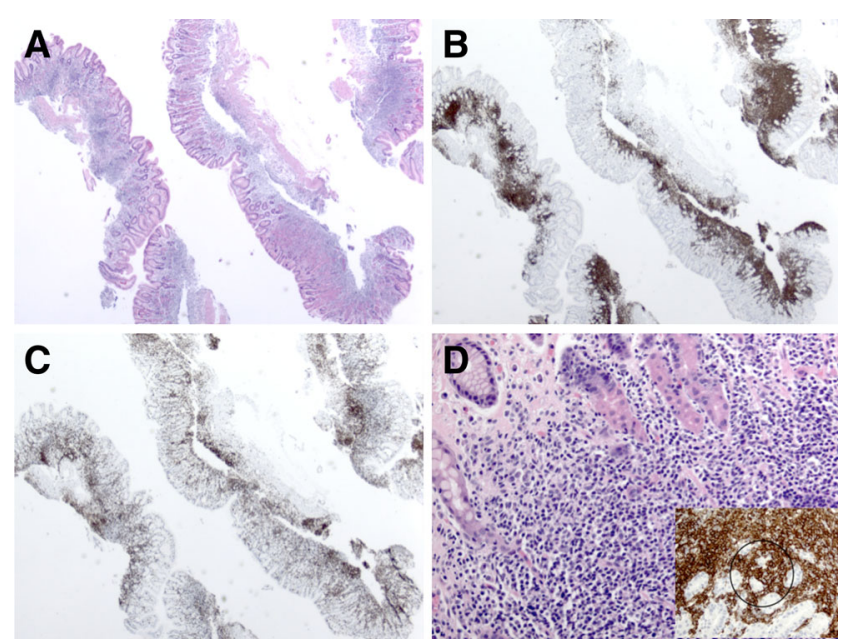

Fig. 4 Morphologic examination of gastric marginal zone lymphoma (case 2 from Table 3). a A moderate to severe lymphocytic infiltrate is seen with a diffuse architectural pattern $(\mathrm{H} \& \mathrm{E}$ stain, $\times 2)$. b CD20 stain highlights numerous B cells $(\times 2)$. c CD3 stain highlights T cells that are much less numerous than the $\mathrm{B}$ cells and less than that seen in reactive gastritis $(\times 2)$. d Lymphocytes have more irregularity to their nuclear membranes, and some have more abundant cytoplasm than seen in reactive gastritis. Lymphoepithelial lesions (LELs) are present $(\mathrm{H} \& \mathrm{E}$, $\times 20$, with insert of CD20 LEL (circle))

\section{Discussion}

Our findings demonstrate that CD10+ clonal (or light chain skewed) B cell populations can be detected by flow cytometry in histologically benign gastric biopsies. Although these cases are not common (only 16 cases were identified during the 14-year period from 2000 to 2014), the true incidence is uncertain, as concurrent flow cytometric studies are not typically performed in patients without a clinical suspicion of gastric lymphoma (samples are taken for flow based on prior history of lymphoma, prior histologic findings worrisome for a lymphiod neoplasm, clinical symptoms, and/or endoscopic/imaging findings). All cases included in this study had been previously evaluated by morphology (some with immunohistochemical studies), and diagnosed as, or favored to be reactive. To verify the original diagnoses, the histological features of each case were independently reviewed by two hematopathologists (L.S. and S.C.), who concurred with the original "reactive" diagnoses. Furthermore, the review of subsequent gastric biopsies (or lack thereof) and the available clinical information demonstrate that none of these patients have developed new and/or recurrent lymphoma (clinical follow-up 2 months to 10 years). Taken together, these findings support the notion that $\mathrm{CD} 10+$ clonal $\mathrm{B}$ cell populations in histologically benign gastric lymphoid proliferations may represent reactive clonal hyperplasia of follicle center B cells rather than an underlying lymphoma, similar to clonal populations that have previously been published in lymph nodes with reactive follicular hyperplasia and in the thyroid in Hashimoto thyroiditis [13, 20]. In fact, this is 
Table 4 Flow cytometric findings of clonal B cell populations in mucosa-associated follicular lymphomas

\begin{tabular}{|c|c|c|c|c|c|c|c|c|c|c|}
\hline Case no. & Age/sex & Biopsy site & Light chain & CD10 & CD19 & $\mathrm{CD} 20$ & CD38 & BCL-2 & $\begin{array}{l}\text { Estimated clonal B } \\
\text { cells ( } \% \text { of B cells) }\end{array}$ & $\begin{array}{l}\text { Estimated clonal B } \\
\text { cells ( } \% \text { of white cells) }\end{array}$ \\
\hline 1 & $66 / \mathrm{M}$ & Duodenum & $\kappa$ & Yes & $\mathrm{Nml}$ & $\mathrm{Nml}$ & NR & $\mathrm{ND}$ & NR & 43 \\
\hline 2 & $50 / \mathrm{F}$ & Duodenum & $\lambda(0.07)$ & Yes & Increased & Increased & $\mathrm{Nml}$ & ND & 92 & 30 \\
\hline 3 & $50 / \mathrm{F}$ & Lung & $\lambda(0.004)$ & Yes & $\mathrm{Nml}$ & Subset decreased & $\mathrm{Nml}$ & ND & 99 & 64 \\
\hline 4 & $60 / \mathrm{M}$ & Parotid & sIg negative & Yes & Decreased & $\mathrm{Nml}$ & $\mathrm{Nml}$ & Pos & 38 & 20 \\
\hline 5 & $87 / F$ & Orbit & sIg negative & Yes & Decreased & $\mathrm{Nml}$ & $\mathrm{Nml}$ & ND & $15^{\mathrm{a}}$ & 8 \\
\hline 6 & $73 / \mathrm{M}$ & Parotid & sIg negative & Yes & Decreased & Decreased & $\mathrm{Nml}$ & ND & $59^{\mathrm{a}}$ & 40 \\
\hline 7 & $61 / \mathrm{M}$ & Orbit & $\lambda$ & Yes & $\mathrm{Nml}$ & Decreased & $\mathrm{Nml}$ & Pos & NR & NR \\
\hline
\end{tabular}

Assessment of kappa/lambda ratio only possible in patients 2 and 3

sIg surface light chain, $N D$ not done, $N R$ not reported, $N m l$ normal or similar to background B cells, Pos positive

${ }^{\text {a }}$ Patient also had concurrent chronic lymphocytic leukemia/small lymphocytic lymphoma

consistent with the previous report that a polyclonal set of B cells, upon activation by antigen, may go through multiple rounds of proliferation and mutation, ultimately leading to a few large dominating B cell clones after elimination of unselected cells [21]. As such, the presence of monoclonal CD10+ B cells identified by flow cytometry should be correlated with morphologic, clinical, and potentially cytogenetic/molecular findings before making a final diagnosis. Although it is unclear as to what antigens are responsible for activating monoclonal $\mathrm{CD} 10+\mathrm{B}$ cells, immunologic response to $H$. pylori infection and/or a prior history of lymphoma may play a role, as approximately $30 \%$ of the cases had one of these potential risk factors.

Features associated more commonly with overt lymphoma in a $\mathrm{CD} 10+$ population include a high percentage of clonal cells (greater than $50 \%$ of all B cells), decreased expression of CD19, and normal CD38 expression (Fig. 1c, d). This is in agreement with the previous reports that FL often aberrantly expresses CD20 and/or CD19 (typically decreased) with normal (or decreased in comparison to follicular hyperplasia) CD38 [19, 22]. By contrast, the reactive clonal B cells of follicular hyperplasia more consistently display slightly increased to increased CD20 and CD38 expression, with normal CD19 (Fig. 1a, 1b), also similar to that previously reported in lymph nodes [14, 17, 18]. The clonal but reactive population in this setting typically represents less than $20 \%$ of all B cells ( $81 \%$ of cases in this study) or less than $10 \%$ of all white cells ( $88 \%$ of cases in this study). Flow cytometric evaluation of BCL-2 overexpression is very useful in such cases, since increased BCL-2 expression is detectable in a majority of FL cases, whereas reactive follicular lymphoid hyperplasia consistently shows negative (or rarely indeterminate) BCL-2 staining by flow cytometry $[2,17,23-26]$. Indeed, the $\mathrm{CD} 10+$ clonal GC B cell populations studied in our series did not overexpress BCL-2 nor have the patients developed lymphoma based on the review of subsequent gastric biopsies and the available clinical information.
Furthermore, the review of morphologic and endoscopic findings demonstrates that the presence of mild to moderate lymphocytic infiltrates having a nodular/lymphoid aggregate architectural pattern and/or the presence of GCs (without evidence of FL in situ) supports a benign diagnosis (Fig. 3a-d), whereas the gastric MALT lymphomas in this study typically showed diffuse, moderate to severe lymphocytic infiltrates with frequent LELs and lack of GCs (Fig. 4a-d) [2, 27]. Although GCs were seen in only $50 \%$ of cases (that would typically correspond to a $\mathrm{CD} 10+\mathrm{B}$ cell population in a benign setting), the findings suggest that there was variation in the cellular components present in the tissue sampled for flow cytometry versus the tissue sampled for H\&E examination (multiple H\&E levels were morphologically evaluated).

In conclusion, the data presented here provides important information regarding the management of $\mathrm{CD} 10+$ clonal $\mathrm{B}$ cell populations identified by flow cytometry and the importance of correlation with the morphologic and clinical findings. The findings support conservative clinical management for patients with $\mathrm{CD} 10+$ clonal $\mathrm{B}$ cell populations identified by flow cytometry but benign histology and reactive rather than aberrant immunophenotypic features, as available clinical follow-up revealed no evidence of lymphoma in all 16 cases. Interesting future directions could include evaluation of the mutational landscape of both reactive and neoplastic gastric processes, as well as evaluation for these clonal (or skewed) $\mathrm{CD} 10+\mathrm{B}$ cell populations in other parts of the gastrointestinal tract.

Compliance with ethical standards All procedures performed in studies involving human participants were in accordance with the ethical standards of the institutional and/or national research committee and with the 1964 Helsinki declaration and its later amendments or comparable ethical standards. For this type of study, formal consent is not required.

Source of funding None declared. 
Conflict of interest The authors declare that they have no conflicts of interest.

\section{References}

1. Hatano B, Ohshima K, Tsuchiya T, Yamaguchi T, Kawasaki C, Kikuchi M (2002) Clinicopathological features of gastric B-cell lymphoma: a series of 317 cases. Pathol Int 52(11):677-682

2. LeBrun DP, Kamel OW, Cleary ML, Dorfman RF, Warnke RA (1992) Follicular lymphomas of the gastrointestinal tract: pathologic features in 31 cases and bcl-2 oncogenic protein expression. Am J Pathol 140(6):1327-1335

3. Shia J, Teruya-Feldstein J, Pan D, Hegde A, Klimstra DS, Chaganti RS, Qin J, Portlock CS, Filippa DA (2002) Primary follicular lymphoma of the gastrointestinal tract: a clinical and pathologic study of 26 cases. Am J Surg Pathol 26(2):216-224

4. Tzankov A, Hittmair A, Müller-Hermelink HK, Rüdiger T, Dirnhofer S (2002) Primary gastric follicular lymphoma with parafollicular monocytoid B-cells and lymphoepithelial lesions, mimicking extranodal marginal zone lymphoma of MALT. Virchows Arch 441(6):614-617

5. Norimura D, Fukuda E, Yamao T, Ozawa E, Sato S, Moriuchi Y, Iwasaki K, Niino D, Matsushima K, Akazawa Y, et al. (2012) Primary gastric follicular lymphoma manifesting as a submucosal tumor-like lesion. Dig Endosc 24(5):389

6. Kaleem Z (2006) Flow cytometric analysis of lymphomas: current status and usefulness. Arch Pathol Lab Med 130(12):1850-1858

7. Kadowaki I, Ichinohasama R, Sasaki O, Kimura J, Kameoka JI, Meguro K, Endo K, Tobinai K, Sasaki T, Sawai T, et al. (1998) Reassessment of non-Hodgkins's lymphoma with a "nodular" growth variant: a clinicopathologic study of follicular, mantle cell and marginal zone lymphomas prospectively diagnosed with multiparameter analyses. Leuk Lymphoma 31(3-4):393-403

8. Yanai S, Nakamura S, Takeshita M, Fujita K, Hirahashi M, Kawasaki K, Kurahara K, Sakai Y, Matsumoto T (2011) Translocation $\mathrm{t}(14 ; 18) / \mathrm{IGH}-\mathrm{BCL} 2$ in gastrointestinal follicular lymphoma: correlation with clinicopathologic features in 48 patients. Cancer 117(11):2467-2477

9. Saxena A, Moshynska O, Kanthan R, Bhutani M, Maksymiuk AW, Lukie BE (2000) Distinct B-cell clonal bands in helicobacter pylori gastritis with lymphoid hyperplasia. J Pathol 190(1):47-54

10. Schulman H, Sickel J, Kleinman MS, Adams JT (1991) Gastric "pseudolymphoma" with restricted light chain expression in a patient with obscure gastrointestinal blood loss. Dig Dis Sci 36(10): 1495-1499

11. Georgopoulos SD, Triantafyllou K, Fameli M, Kitsanta P, Spiliadi C, Anagnostou D, Ladas SD (2005) Molecular analysis of B-cell clonality in helicobacter pylori gastritis. Dig Dis Sci 50(9):16161620

12. Hummel M, Oeschger S, Barth TF, Loddenkemper C, Cogliatti SB, Marx A, Wacker HH, Feller AC, Bernd HW, Hansmann ML, et al. (2006) Wotherspoon criteria combined with B cell clonality analysis by advanced polymerase chain reaction technology discriminates covert gastric marginal zone lymphoma from chronic gastritis. Gut 55(6):782-787

13. Kussick SJ, Kalnoski M, Braziel RM, Wood BL (2004) Prominent clonal B-cell populations identified by flow cytometry in histologically reactive lymphoid proliferations. Am J Clin Pathol 121(4): 464-472

14. Reichard KK, McKenna RW, Kroft SH (2003) Comparative analysis of light chain expression in germinal center cells and mantle cells of reactive lymphoid tissues. A four-color flow cytometric study. Am J Clin Pathol 119(1):130-136

15. Knowles DM (2001) Immumophenotypic markers useful in the diagnosis and classification of hematopoietic neoplasms. In: Knowles DM (ed) Neoplastic hematopathology, 2nd edn. Lippincott Williams \& Wilkins, Philadelphia, p. 185

16. Zhao XF, Cherian S, Sargent R, Seethala R, Bonner H, Greenberg B, Bagg A (2005) Expanded populations of surface membrane immunoglobulin light chain-negative B cells in lymph nodes are not always indicative of B-cell lymphoma. Am J Clin Pathol 124(1):143-150

17. Cornfield DB, Mitchell DM, Almasri NM, Anderson JB, Ahrens KP, Dooley EO, Braylan RC (2000) Follicular lymphoma can be distinguished from benign follicular hyperplasia by flow cytometry using simultaneous staining of cytoplasmic bcl-2 and cell surface CD20. Am J Clin Pathol 114(2):258-263

18. Mantei K, Wood BL (2009) Flow cytometric evaluation of CD38 expression assists in distinguishing follicular hyperplasia from follicular lymphoma. Cytometry B Clin Cytom 76(5):315-320

19. Ray S, Craig FE, Swerdlow SH (2005) Abnormal patterns of antigenic expression in follicular lymphoma: a flow cytometric study. Am J Clin Pathol 124(4):576-583

20. Chen HI, Akpolat I, Mody DR, Lopez-Terrada D, De Leon AP, Luo Y, Jorgensen J, Schwartz MR, Chang CC (2006) Restricted kappa/ lambda light chain ratio by flow cytometry in germinal center B cells in Hashimoto thyroiditis. Am J Clin Pathol 125(1):42-48

21. Küppers R, Zhao M, Hansmann ML, Rajewsky K (1993) Tracing B cell development in human germinal centres by molecular analysis of single cells picked from histological sections. EMBO J 12(13): 4955-4967

22. Bellido M, Rubiol E, Ubeda J, López O, Estivill C, Carnicer M, Muñoz L, Bordes R, Sierra J, Nomdedéu J (2001) Flow cytometry using the monoclonal antibody $\mathrm{CD} 10-\mathrm{Pe} / \mathrm{Cy} 5$ is a useful tool to identify follicular lymphoma cells. Eur J Haematol 66(2):100-106

23. Ngan BY, Chen-Levy Z, Weiss LM, Warnke RA, Cleary ML (1988) Expression in non-Hodgkin's lymphoma of the bcl-2 protein associated with the $\mathrm{t}(14 ; 18)$ chromosomal translocation. N Engl J Med 318(25):1638-1644

24. Pezzella F, Tse AG, Cordell JL, Pulford KA, Gatter KC, Mason DY (1990) Expression of the bcl-2 oncogene protein is not specific for the 14;18 chromosomal translocation. Am J Pathol 137(2):225-232

25. Zutter M, Hockenbery D, Silverman GA, Korsmeyer SJ (1991) Immunolocalization of the bcl-2 protein within hematopoietic neoplasms. Blood 78(4):1062-1068

26. Cook JR, Craig FE, Swerdlow SH (2003) Bcl-2 expression by multicolor flow cytometric analysis assists in the diagnosis of follicular lymphoma in lymph node and bone marrow. Am J Clin Pathol 119(1):145-151

27. Freeman C, Berg JW, Cutler SJ (1972) Occurrence and prognosis of extranodal lymphomas. Cancer 29(1):252-260 